\title{
Angular correlations of identified particles in the STAR BES data
}

\author{
Andrzej Lipiec*, for the STAR Collaboration \\ Warsaw University of Technology \\ E-mail: andrew.lipiec@gmail.com
}

The angular correlation function $(\mathrm{CF})$ in this analysis refers to the correlation of particles in the relative pseudorapidity and relative azimuthal angle. It is used to study properties of strongly interacting matter at relativistic energies. Recent results from the ALICE experiment at LHC show unexpected structures of $\mathrm{CF}$ in the proton-proton and antiproton-antiproton correlations. Also, results from the STAR experiment at RHIC on directed flow $\left(d v_{1} / d y\right)$ for the net-baryons are intriguing.

The STAR capability of particle identification at midrapidity, paired with the data from broad energy range of Au+Au collisions in the Beam Energy Scan program, provides unique opportunity to investigate the phase diagram of strongly interacting matter through the $\mathrm{CF}$ analysis. In this talk, recent STAR experimental results from the $\mathrm{Au}+\mathrm{Au}$ collisions at $\sqrt{{s_{N N}}}=19.6 \mathrm{GeV}$ from the RHIC's Beam Energy Scan will be presented.

The European Physical Society Conference on High Energy Physics

5-12 July

Venice, Italy

${ }^{*}$ Speaker. 


\section{Introduction}

The Beam Energy Scan (BES) program was carried out by the STAR experiment (Solenoidal Tracker At RHIC) at Relativistic Heavy Ion Collider (RHIC) in years 2010, 2011 and 2014, to collect experimental data for studying phase diagram of strongly interacting matter. STAR experiment recorded $\mathrm{Au}+\mathrm{Au}$ collisions at $\sqrt{s_{N N}}=7.7,11.5,14.5,19.6,27,39$ and $62.4 \mathrm{GeV}$. These datasets allow to search for the turn-off of Quark Gluon Plasma signatures, the signals of phase transition, phase boundary, and evidence for the critical point existence. The STAR experiment has already published a number of results from the BES data (for example [1], [2]). In this paper, we present the study of two-identified-particle correlations in $\mathrm{Au}+\mathrm{Au}$ collisions at $\sqrt{s_{N N}}=19.6 \mathrm{GeV}$.

The two-particle correlations are studied to extract properties of the medium created in highenergy collisions. The recent findings show intriguing structures in angular correlation function, i.e. the correlations of particles with respect to their pseudorapidity difference $(\Delta \eta)$ and relative azimuthal angle $(\Delta \phi)$. The ALICE experiment recently reported an anti-correlation of two protons in $\mathrm{p}+\mathrm{p}$ collisions at $\sqrt{s}=7 \mathrm{TeV}$, which is not seen in two-pion and two-kaon correlations [3]. Furthermore, the STAR experiment observed similar anti-correlation of two protons in the 0-5\% central Au+Au collisions at $\sqrt{s_{N N}}=7.7,11.5,14.5,19.6,27,39,62.4$ and $200 \mathrm{GeV}$ [4].

In this proceeding, the latest STAR measurements of angular correlation function for protons and pions in $\mathrm{Au}+\mathrm{Au}$ at $\sqrt{s_{N N}}=19.6 \mathrm{GeV}$ is presented. The previous angular correlations analysis carried out by the STAR experiment was limited to $|\Delta \eta| \leq 1$. This study extends the analyzed $\Delta \eta$ range up to \pm 2 units. The paper covers results from different centrality classes $(70-80 \%$, $60-70 \%, 50-60 \%, 40-50 \%, 30-40 \%, 20-30 \%, 10-20 \%, 5-10 \%$, and 0-5\%) of Au+Au collisions at $\sqrt{s_{N N}}=19.6 \mathrm{GeV}$. The future extension of this study to other BES energies may allow the disentanglement of sources of correlations.

\section{Analysis method}

The angular correlation function is defined (following ref. [5]) as:

$$
\frac{\Delta \rho_{s}}{\sqrt{\rho}}=\frac{N_{s}}{\Delta \eta \Delta \phi} \cdot\left(r_{s}-1\right),
$$

where $N_{s}$ is the mean particle multiplicity in the STAR acceptance, $\Delta \eta=2, \Delta \phi=2 \pi$, and $r_{s}$ is a ratio of particle pair density in the single event divided by the particle pairs density obtained from mixed events. The subscript $s$ denote particle specie (in case of this study either pions or protons).

The protons and pions were identified by the ionization energy loss $(\mathrm{dE} / \mathrm{dx})$ in Time Projection Chamber (TPC). The measured $\mathrm{dE} / \mathrm{dx}$ value had to fall within two standard deviations from the theoretical value for a given particle type and had to be further than 3 standard deviations from theoretical values for other particle species at given momentum. The TPC signal alone allows to utilize STAR acceptance and to identify pions and protons up to $p=0.8 \mathrm{GeV} / \mathrm{c}$. That imposes cut on particle momentum: $p=(0.2-0.8) \mathrm{GeV} / \mathrm{c}$, and pseudorapidity: $|\eta| \leq 1$. Analyzed Au+Au collisions at $\sqrt{s_{N N}}=19.6 \mathrm{GeV}$ were divided into 9 centrality classes based on number of charged particles within $|\eta| \leq 1$. The statistical background was estimated by the event mixing technique, i.e. by correlating particles from the analyzed event with particles from the other event. For each 
analyzed event 5 events were used for background estimation. Only similar events that did not differ from the analyzed event more than $2 \mathrm{~cm}$ in $V_{Z}$ (interaction point position along the beam axis) and not more than 40 charged tracks multiplicity were used for event mixing.

\section{Results and discussion}

Figure 1 shows STAR results on angular correlation function for combined proton-proton and antiproton-antiproton pairs, for all measured centrality classes of $\mathrm{Au}+\mathrm{Au}$ at $\sqrt{s_{N N}}=19.6 \mathrm{GeV}$. In two-proton correlations, one can observe an anti-correlation ${ }^{1}$ with the minimum around $(\Delta \eta, \Delta \phi) \approx(0,0)$, which overlaps with the sharp peak centered at the same point - most likely effect of strong interactions between protons. The sharp peak fades away with more central collisions. This can be interpreted as that the system size is larger in more central collisions. The anti-correlation is present in all centrality classes of $\mathrm{Au}+\mathrm{Au}$ at $\sqrt{s_{N N}}=19.6 \mathrm{GeV}$ and resembles what was observed by ALICE in $\mathrm{p}+\mathrm{p}$ at $\sqrt{s}=7 \mathrm{TeV}$ [3] and by STAR in 0-5\% Au+Au at all BES beam energies [4]. The disentanglement of the sharp peak and anti-correlation structures will allow to quantify the strength of anti-correlation.

Figure 2 shows the comparison of STAR results on angular correlation function for $p p$ and $\bar{p} \bar{p}$ pairs (left) and like-sign pions (right), for three selected centrality classes of $\mathrm{Au}+\mathrm{Au}$ at $\sqrt{s_{N N}}=19.6 \mathrm{GeV}: 70-60 \%$ (top), 40-30\% (middle), and 0-5\% (bottom). The results for $p p$ and $\bar{p} \bar{p}$ differ significantly from correlations of like-sign pions. The ridge at $\Delta \phi=0$ and $\Delta \phi=\pi$ in two-pion correlations is the strongest in mid-central collisions, which suggests that elliptic flow $\left(v_{2}\right)$ might be a source of such a correlation. In two-proton distributions, the effect of $v_{2}$ is not clearly visible due to the statistical noise coupled with the broad anti-correlation at $\Delta \phi=0$.

We also report that anti-correlation observed for proton-antiproton pairs differs from the one observed for $p p$ and $\bar{p} \bar{p}$ pairs. Figure 3 shows results on angular correlation function of protonantiproton pairs at $\sqrt{s_{N N}}=19.6 \mathrm{GeV}$. The anti-correlation centered at $(\Delta \eta, \Delta \phi \approx(0,0))$ differs from the one observed for $p p$ and $\bar{p} \bar{p}$ pairs. In proton-antiproton case the amplitude is larger. Both amplitude and width do not change significantly for different centrality classes. One of possible explanations for this discrepancy is that proton-antiproton correlations may involve annihilation processes.

\section{Summarry}

The STAR results on angular correlations of identified hadrons in $\mathrm{Au}+\mathrm{Au}$ collisions at $\sqrt{s_{N N}}=19.6 \mathrm{GeV}$ were presented. Results for two-pion correlations are consistent with the previous measurements in $\mathrm{Au}+\mathrm{Au}$ at $200 \mathrm{GeV}$ [6]. Correlations of proton-antiproton pairs show clear, strong anti-correlations near $(\Delta \eta ; \Delta \phi) \approx(0 ; 0)$. It is possible that such a shape might be caused by annihilation processes.

The broad anti-correlation in $p p$ and $\bar{p} \bar{p}$ pairs is visible in all centrality classes of $\mathrm{Au}+\mathrm{Au}$ collisions at $\sqrt{s_{N N}}=19.6 \mathrm{GeV}$. Similar anti-correlation was observed in $0-5 \%$ central $\mathrm{Au}+\mathrm{Au}$

\footnotetext{
${ }^{1}$ In this paper every significant depletion in $\mathrm{CF}$ is considered an anti-correlation. The value of $\mathrm{CF}$ does not have to be below 0 , as it is not corrected for a constant offset present in each $\Delta \eta \Delta \phi$ bin. The offset is caused by the event-by-event charged particle multiplicity fluctuations
} 
collision at all BES energies [4] and resembles ALICE results from $\mathrm{p}+\mathrm{p}$ at $\sqrt{s}=7 \mathrm{TeV}$ collisions [3]. This anti-correlation seems to be: a) collision energy independent, b) system independent, and c) system size and geometry independent. Further study of other BES energies with the same approach might provide a better understanding of interactions between protons and will allow for disentanglement of sources of correlations.

\section{Acknowledgements}

This work is funded by the National Science Centre (Poland) within the scope of project number: UMO-2016/21/N/ST2/00315

\section{References}

[1] L. Adamczyk et al. (STAR Collaboration), Beam-Energy Dependence of the Directed Flow of Protons, Antiprotons, and Pions in Au+Au Collisions, Phys.Rev.Lett. 112 (2014)

[2] L. Adamczyk et al. (STAR Collaboration), Energy Dependence of Moments of Net-proton Multiplicity Distributions at RHIC, Phys. Rev. Lett. 112 (2014)

[3] L. K. Graczykowski, M. A. Janik, ALICE Collaboration, Angular correlations of identified charged particles measured in pp collisions by ALICE at the LHC, Nucl. Phys. A 926 (2014)

[4] S. Jowzaee, STAR Collaboration, Rapidity correlations in the RHIC Beam Energy Scan, https://arxiv.org/pdf/1708.03364.pdf

[5] G. Agakishiev et al. (STAR Collaboration),Anomalous centrality evolution of two-particle angular correlations from Au-Au collisions at $\sqrt{s_{N N}}=62$ and $200 \mathrm{GeV}$, Phys.Rev. C 86 (2012) 064902

[6] P. Bhattarai, Two-particle Correlations of Identified Particles in Heavy Ion Collisions at STAR, Doctoral Dissertation (2016). 
U $\times 10^{-3}$

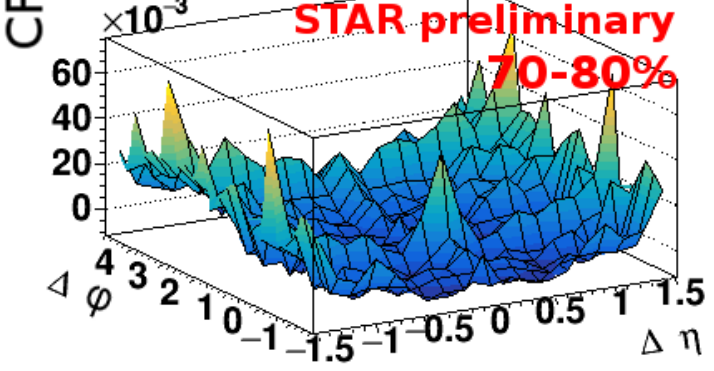

山 (c)

U $\times 10^{-3} \quad$ STAR pretiminary

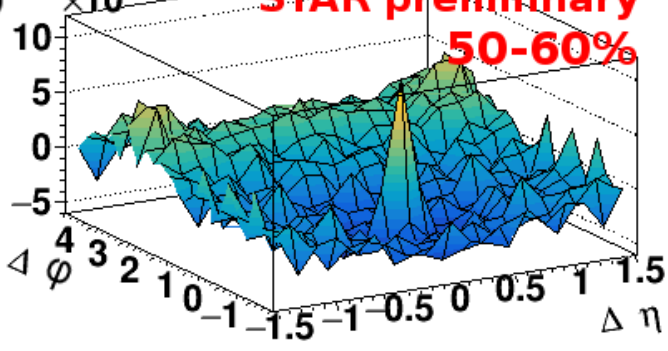

㟧 (e)

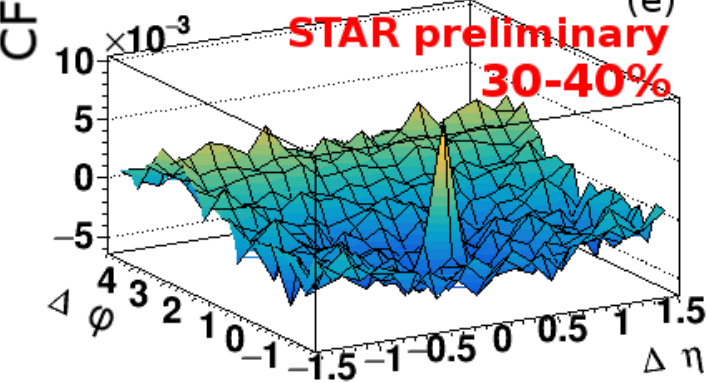

(b)

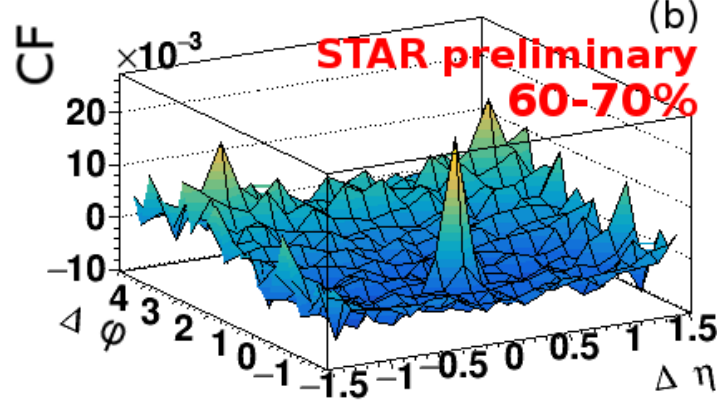

(d)

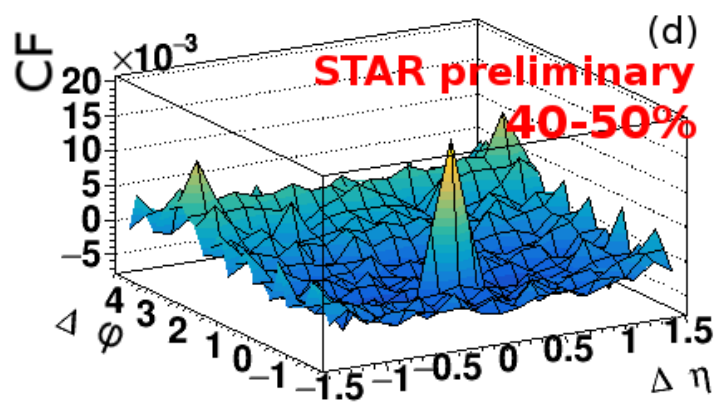

(f)

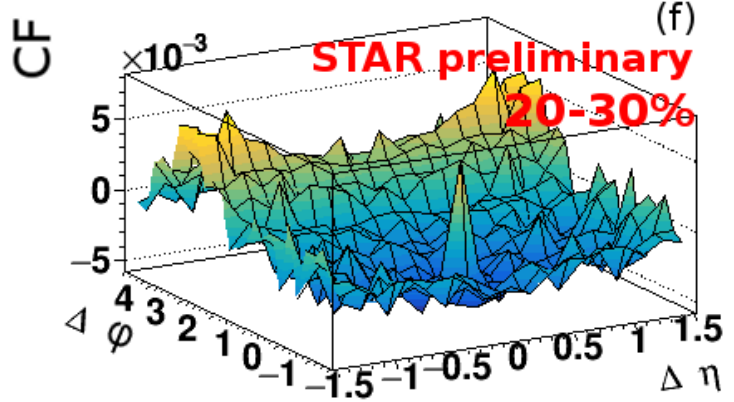

(h)

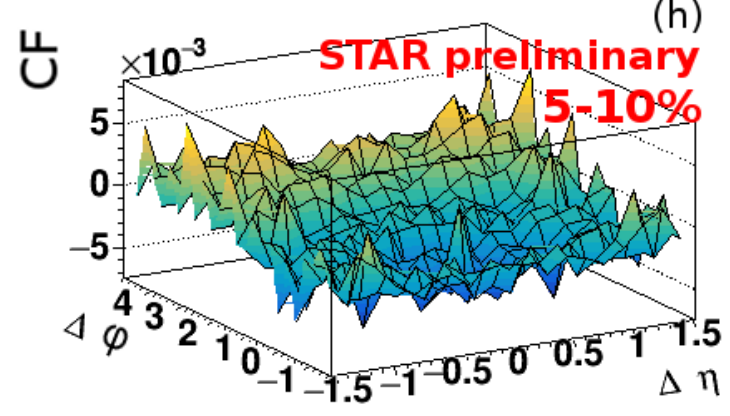

(i)

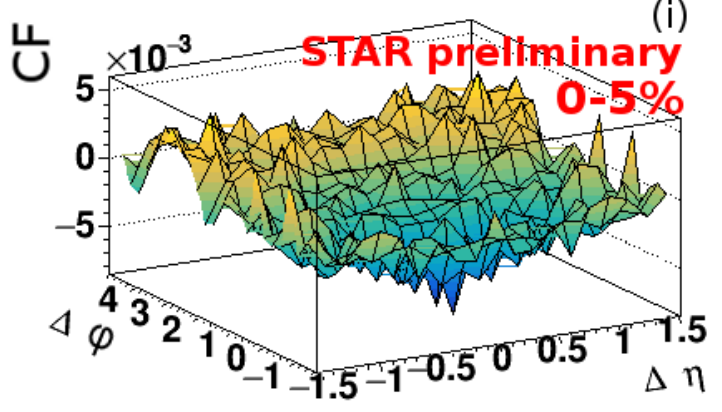

4

Figure 1: Figure shows $\Delta \eta, \Delta \phi$ dependence of correlation function for $p p$ and $\bar{p} \bar{p}$ pairs, obtained by STAR experiment. Subsequent panels correspond to centrality classes of Au+Au collisions at $\sqrt{s_{N N}}=19.6 \mathrm{GeV}$ : $80-70 \%$ (a), $60-70 \%$ (b), $50-60 \%$ (c), $40-50 \%$ (d), $30-40 \%$ (e), $20-30 \%$ (f), $10-20 \%$ (g), $5-10 \%$ (h), $0-5 \%$ (i). Note the different scale on vertical axis. 

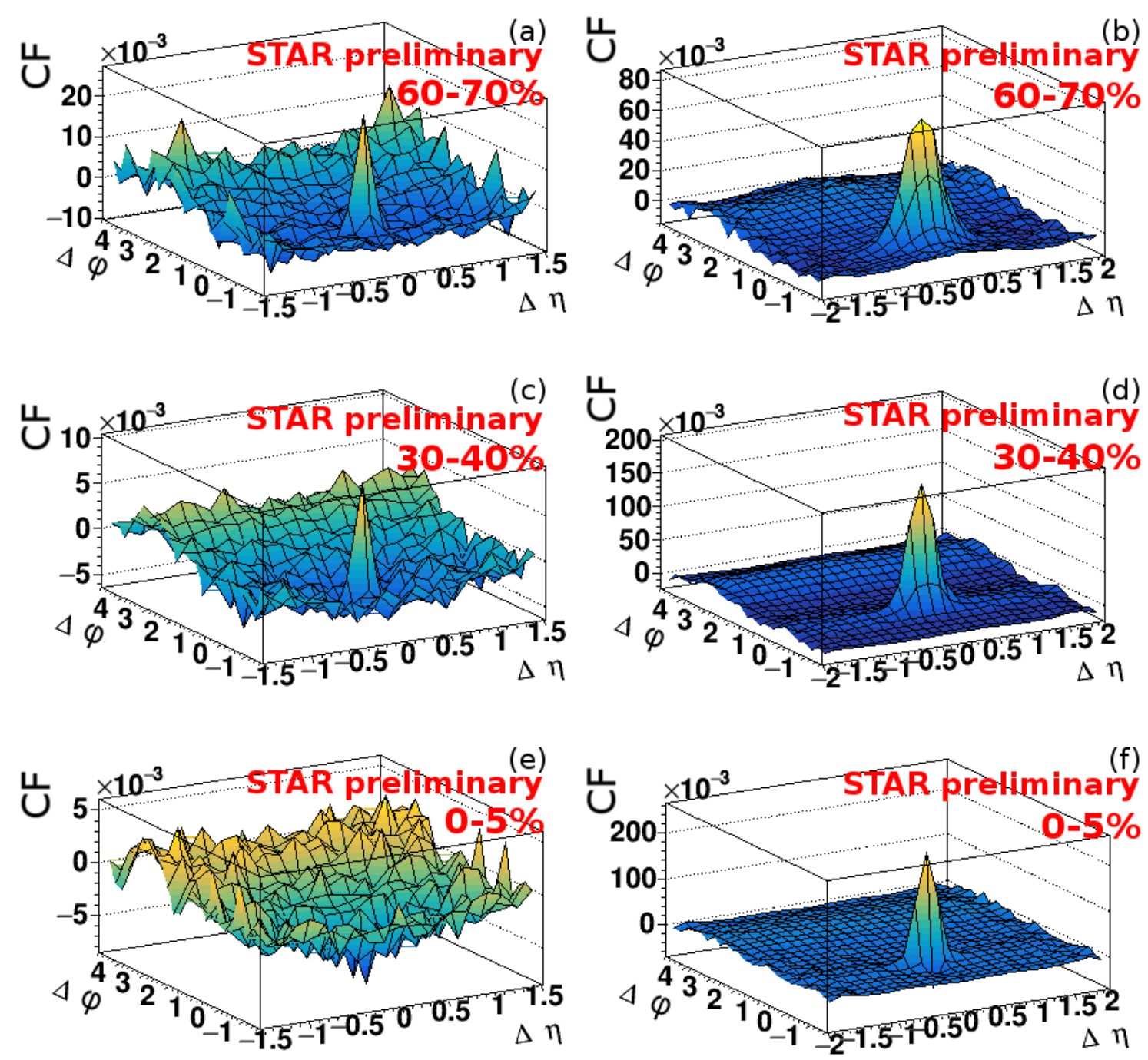

Figure 2: Figure shows $\Delta \eta, \Delta \phi$ dependence of correlation function for $p p$ and $\bar{p} \bar{p}$ pairs, [panels (a), (c), (e)] and two like-sign pions [panels (b), (d), (f)] obtained by STAR experiment. Subsequent rows correspond to selected centrality classes of Au+Au collisions at $\sqrt{s_{N N}}=19.6 \mathrm{GeV}: 60-70 \%$ [(a) and (b)], 30-40\% [(c) and (d)], and 0-5\% [(e) and (f)].

(a) STAR pieliminary (b)
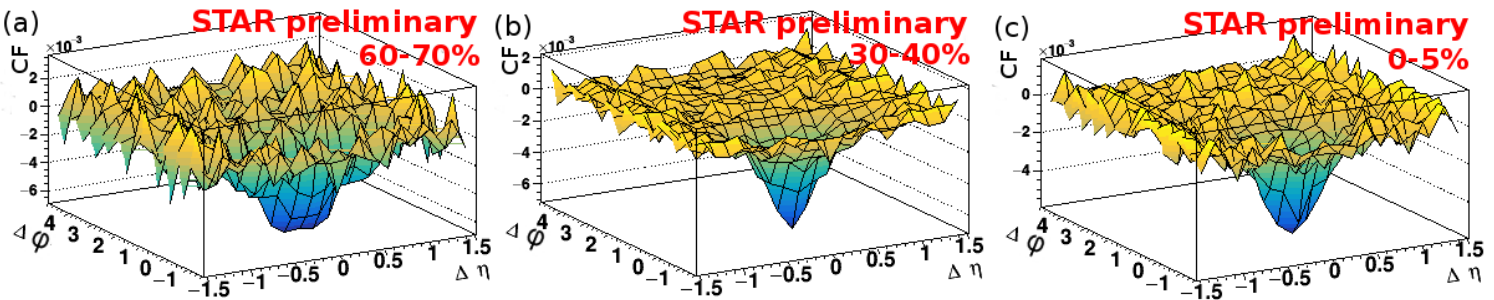

Figure 3: Figure shows $\Delta \eta, \Delta \phi$ dependence of correlation function for proton-antiproton pairs, obtained by STAR experiment. Subsequent columns correspond to selected centrality classes of Au+Au collisions at $\sqrt{s_{N N}}=19.6 \mathrm{GeV}: 60-70 \%$ is shown in panel (a), 30-40\% in (b), and 0-5\% in (c). 